\title{
THE MONITORING CASE OF GROUND-BASED SYNTHETIC APERTURE RADAR WITH FREQUENCY MODULATED CONTINUOUS WAVE SYSTEM
}

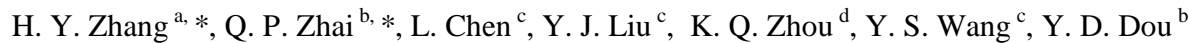 \\ ${ }^{a}$ School of Naval Architecture and Mechanical-electrical Engineering, Zhejiang ocean university, Zhoushan, China - \\ haoyu19871202@163.com \\ b Tianjin Saruide Technology Co., Ltd, Tianjin, China - zhai_qiuping@ tripleradar.cn \\ ${ }^{c}$ Beijing Five Star Electronic Technology(Beijing) Co., Ltd, Beijing, China - idowman@ge.ucl.ac.uk \\ ${ }^{\mathrm{d}}$ Shool of Geomatics and Urban Spatial Information, Beijing University of Civil Engineering and Architecture, Beijing, China - \\ zhoukeqin@bucea.edu.cn
}

Working Group III/2

KEY WORDS: Landslide Monitoring, Synthetic Aperture Radar, High-speed Data Acquisition, FMCW, Measured Data, Severe Weather

\begin{abstract}
:
The features of the landslide geological disaster are wide distribution, variety, high frequency, high intensity, destructive and so on. It has become a natural disaster with harmful and wide range of influence. The technology of ground-based synthetic aperture radar is a novel deformation monitoring technology developed in recent years. The features of the technology are large monitoring area, high accuracy, long distance without contact and so on. In this paper, fast ground-based synthetic aperture radar (Fast-GBSAR) based on frequency modulated continuous wave (FMCW) system is used to collect the data of Ma Liuzui landslide in Chongqing. The device can reduce the atmospheric errors caused by rapidly changing environment. The landslide deformation can be monitored in severe weather conditions (for example, fog) by Fast-GBSAR with acquisition speed up to 5 seconds per time. The data of Ma Liuzui landslide in Chongqing are analyzed in this paper. The result verifies that the device can monitor landslide deformation under severe weather conditions.
\end{abstract}

\section{INTRODUCTION}

The ground-based synthetic aperture radar (GB-SAR) is a novel deformation monitoring technology developed in recent years. It can transmit/receive electromagnetic waves and obtain the displacement information in the monitoring area based on interference technology. The GB-SAR is capable of monitoring large area in a long range without contact (Wang and Zhou, 2012). The traditional pulsed SAR compresses the transmitted pulses to reach a high resolution image. However, the pulse SAR needs to transmit high-energy pulse in a very short time period, with the disadvantages being such as large size, high power consumption, high cost and heavy weight. These disadvantages limit the use of GB-SAR in civilian areas such as emergency rescue and disaster relief.

In this paper, Fast-GBSAR is used to collect the data of the landslide. This Fast-GBSAR combines the FMCW, SAR and interference technologies to monitor high-precision, real-time, large area terrain micro-deformation. The Fast-GBSAR has the advantages of high-speed data acquisition, small volume, light weight, low power consumption, all-day, all-weather and long monitoring distance. In this paper, the experimental results of Ma Liuzui landslide in Chongqing are analysed.

\section{FAST-GBSAR LANDSLIDE MONITORING SITE}

\subsection{System Introduction}

This Fast-GBSAR is the first fully polarized ground based synthetic aperture radar (GB-SAR) in the world based on the advanced FMCW technology. The Fast-GBSAR has two operating modes: RAR mode (Real Aperture Radar) and SAR mode (Synthetic Aperture Radar). SAR mode can obtain both range resolution and azimuth resolution. The SAR mode is used for monitoring regional structures such as open-pit mine, dam, landslide, etc. In this paper, SAR mode is used for monitoring the landslide. The Fast-GBSAR works in the Ku band, as shown in Figure 1, and the specific technical parameters are shown in table 1. The Fast-GBSAR has four antennas and works in full polarization mode $(\mathrm{HH}, \mathrm{VV}, \mathrm{HV}, \mathrm{VH})$. Two antenna configurations is another alternative choice. In this configuration, one antenna transmits signal and both these two antennas receive the reflected signal at the same time. Then the interference process is carried out to calculate the deformation. In this paper, the two antenna configuration is chosen for interference deformation measurement (Figure 3).

* Corresponding author 


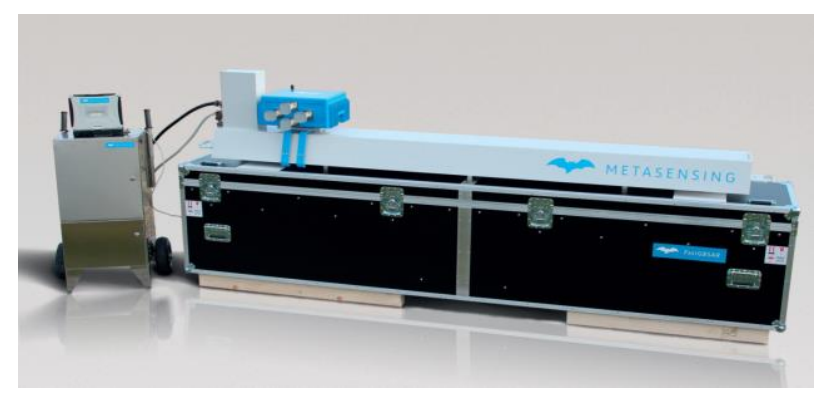

Figure 1. Fast-GBSAR system

\begin{tabular}{|l|c|c|}
\hline Operation Mode & SAR & \multicolumn{1}{c|}{ RAR } \\
\hline Operating Frequency & \multicolumn{2}{|c|}{$17.2 \mathrm{GH}$} \\
Range Resolution & \multicolumn{2}{|c|}{$0.5 \mathrm{~m}$} \\
Maximum Range & \multicolumn{2}{|c|}{$4 \mathrm{Km}$} \\
EIRP Power & \multicolumn{2}{|c|}{$42 \mathrm{dBm}$} \\
Operating temperature & \multicolumn{2}{|c|}{$-20^{\circ} \mathrm{C} \sim 60^{\circ} \mathrm{C}$} \\
Environmental level & \multicolumn{2}{|c|}{ IP65 } \\
Host weight & \multicolumn{2}{|c|}{$10 \mathrm{Kg}$} \\
\hline Precision & $\pm 0.1 \mathrm{~mm}$ & $\pm 0.01 \mathrm{~mm}$ \\
Azimuth Resolution & $4.5 \mathrm{mrad}$ & - \\
Acquisition Time & $5 \mathrm{~s}$ & $0.25 \mathrm{~ms}$ \\
Power Consumption & $<200 \mathrm{~W}$ & $70 \mathrm{~W}$ \\
Linear rail weight & $72 \mathrm{Kg}$ & - \\
Total length of the linear rail & $2606 \mathrm{~mm}$ & - \\
Effective length of the linear rail & $1800 \mathrm{~mm}$ & - \\
\hline
\end{tabular}

Table 1. Fast-GBSAR specific technical parameters

\subsection{The Experimental Setup}

Ma Liuzui landslide is monitored in January 31, 2017. The Ma Liuzui landslide is located in Ma Liuzui town, Banan district, Chongqing city, which is in the southeast of Yangtze river. After the fieldwork is finished, the GB-SAR is mounted on the side of the Yangtze River with the monitoring target on the other side (Figure 2). The monitoring site is carefully chosen with little obstructions. There is thick fog during the day of experiment. The GB-SAR is about $2 \mathrm{~km}$ away from the landslide beyond the scope of human vision.

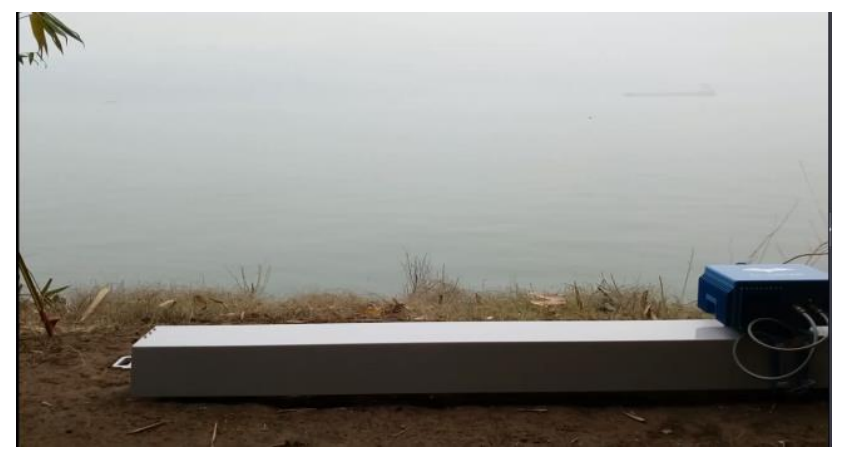

Figure 2. The experimental environment

\subsection{Data Acquisition}

The laptop is connected to the Fast-GBSAR host wifi (the previous ground-based SAR is usually connected to the laptop through the USB serial port, such as Italy's IBIS) after the erection of the system. After the connection is finished, data acquisition process can be controlled by the Fast-GBSAR Controller software in the laptop. It includes experiment time, slide speed of rail, etc. The scene after the erection of the system is shown in Figure 3. The monitoring time of the Ma Liuzui landslide lasts 26 minutes, from: 15:43:38 to 16:09:38.

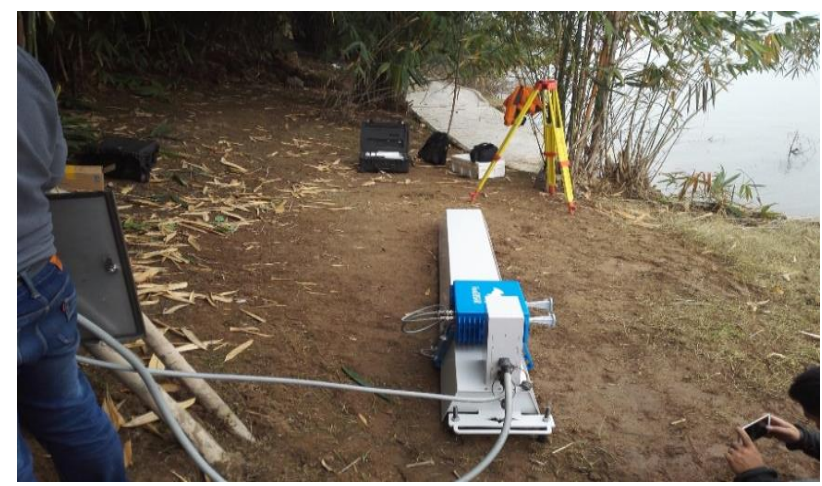

Figure 3. System erection site

\section{TECHNICAL BACKGROUND}

\subsection{The FMCW Based Synthetic Aperture Radar Technology}

The FMCW SAR is a high resolution imaging radar, which is suitable for the applications of miniaturized SAR (Qu and Wang, 2008). The used modulating continuous wave signal is difficult to be detected by the traditional reconnaissance aircraft with large time-bandwidth product. Synthetic Aperture Radar (SAR) is a high-resolution active microwave remote sensing imaging system. SAR has become a very important remote sensing tool, which plays an important role in many fields, such as regional surveillance, terrain mapping, military reconnaissance, and weapons guidance (Jiang et al., 2008).

3.1.1 Signal Model: The synthetic aperture radar with FMCW signal model is analysed in details (Meta et al., 2003), and the zero intermediate frequency signal is obtained by mixing the echo signal directly with the replica of the transmitted signal.

$$
\begin{gathered}
s s\left(t_{r}, t_{a} ; r_{0}\right)=C \\
\exp \left[j 2 \pi f_{c}\left[\mu\left(t_{r}+t_{a}-\tau_{0}\right)+\frac{2 V^{2} t_{a}^{2}}{c_{0} r_{0}}\right]\right] \\
\exp \left[j \pi k_{r}\left[\mu\left(t_{r}+t_{a}-\tau_{0}\right)+\frac{2 V^{2} t_{a}^{2}}{c_{0} r_{0}}-t_{a}\right]^{2}\right]
\end{gathered}
$$

where

$f_{c}=$ signal carrier frequency

$k_{r}=$ signal frequency modulation slope

$V=$ antenna movement speed

$c_{0}=$ speed of light

$r_{0}=$ the minimum distance between antenna and target

$C=$ complex constant

$t_{r}=$ time in distance

$t_{a}=$ time in azimuth 


$$
\begin{aligned}
\tau_{0} & =\frac{2}{c_{0}} \sqrt{r_{0}^{2}+V^{2} t_{a}^{2}} \\
\mu & =1-\frac{2 V^{2} t_{a}}{c_{0} r_{0}}
\end{aligned}
$$

\subsection{Introduction of Ground-based SAR Technology}

The ground-based SAR obtains data through the antenna moving in the horizontal rail with forming the synthetic aperture in azimuth ( $\mathrm{Qu}$ et al., 2011). The time series interference data which are obtained by repeating observations of the target area at different time points is used for the deformation monitoring (Qu et al., 2011). The results of the deformation monitoring can be achieved with the accuracy of millimetre-level. It is a technical means for the deformation monitoring of local area. The GB-SAR can continue to monitor during all-weather condition, while the non-contact capability can obtain the deformation information within the safe distance. Furthermore, the capability of large area monitoring is more conducive to disaster understanding and prediction than the single point deformation monitoring (for example, GPS).

Technical parameters of Fast-GBSAR used in experiment: the 2-D image with $0.75 \mathrm{~m}$ spatial resolution in range and $4.5 \mathrm{mrad}$ in azimuth is generated, while the azimuth resolution is achieved by moving the GB-SAR in the $1.8 \mathrm{~m}$ long rail. For each 5 second, one complex image can be created and used for deformation monitoring, with submillimetre level accuracy.

\subsection{Principle of Interference Technology}

InSAR uses two microwave images of the same target obtained by two separated antennas to obtain elevation information by interferometric technology. Unlike conventional stereoscopic photogrammetry, the received complex images include both amplitude and phase information. The interference image can be created by conjugating the received two complex images as long as they are coherent. By processing the interference images, the corrected phase of each point can be calculated. Then the phase can be used to calculate the slope and elevation of the ground point (Wang et al., 1997). The ground-based SAR interference measurement flow chart is shown in Figure 4.

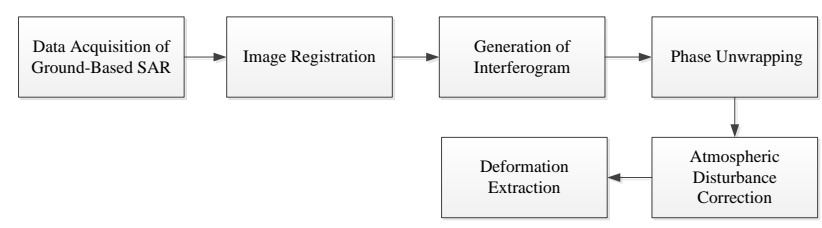

Figure 4. The ground-based SAR interference measurement flow chart

\section{LANDSLIDE MONITORING DATA ANALYSIS}

The received landslide signals by Fast-GBSAR are processed by SePSI software. The distance between the Fast-GBSAR and the mountain is around 2000 meters.

The average amplitude intensity collected from Ma Liuzui landslide is shown in Figure 5. The profile and position of the Ma Liuzhuang landslide can be seen through average intensity. The PS points are chosen in the area where the average intensity is strong. The amplitude dispersion index of Ma Liuzhuang landslide data is shown in Figure 6. The high quality PS points can be obtained by combining the mean amplitude intensity in Figure 4 and the amplitude dispersion index in Figure 6 with applicable threshold. It can be seen that the point of large radar scattering intensity is relatively stable, usually as a PS point through Figure 5 and Figure 6 . The PS points based on the amplitude deviation threshold algorithm mentioned earlier are shown in Figure 7, where the black points are the PS points studied in the experiment. The interference phase is wrapped, and phase unwrapping process is required to calculate the real phase difference. PS triangular mesh created in the phase unwrapping process is shown in Figure 8. Another required step is the atmospheric error correction, in order to ensure the accuracy of the landslide deformation. This step has been done as shown in Figure 9. The final PS points deformation after phase unwrapping and atmospheric phase correction are shown in Figure 10. As seen, the Fast-GBSAR system can effectively measure the deformation of the Liuzhuang landslide under severe weather conditions with submillimetre level accuracy. In Figure 10, the two strongest deformative PS points $(x=-104.7$, $y=1759 ; \mathrm{x}=479.2, \mathrm{y}=1871.6)$ are detected at the top of the Ma Liuzui mountain.

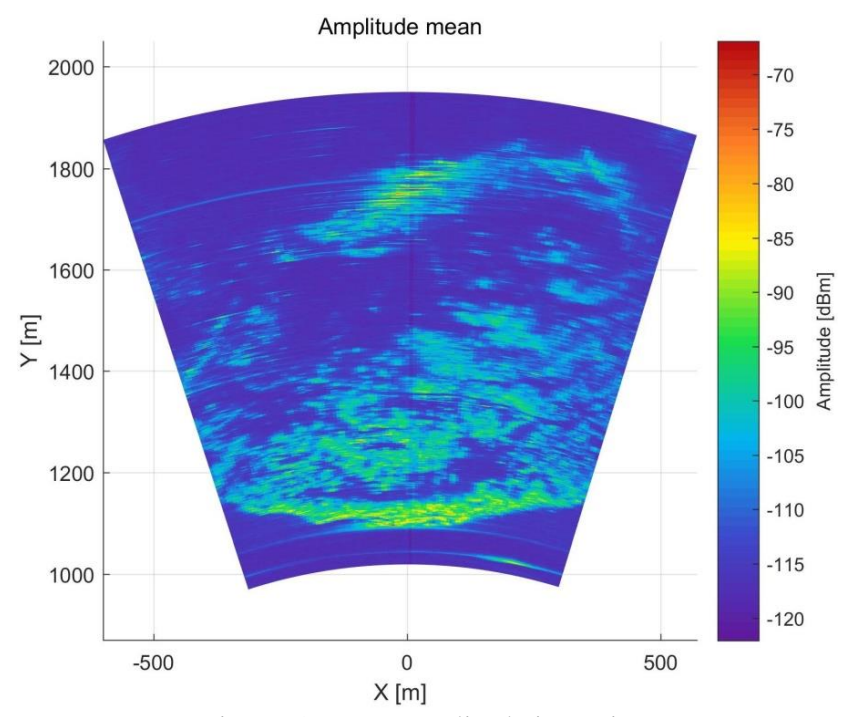

Figure 5. Mean amplitude intensity

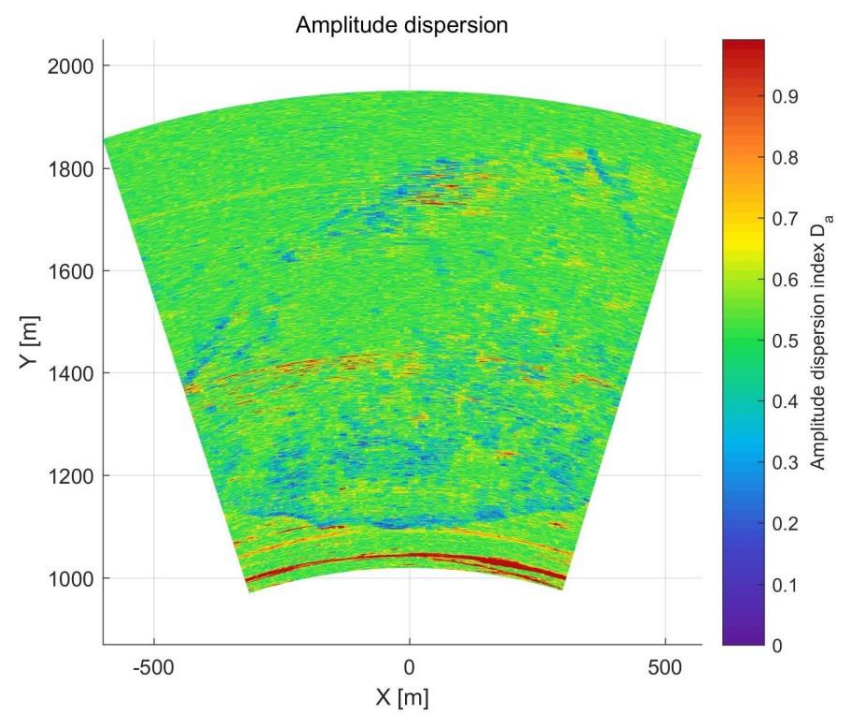

Figure 6. Amplitude dispersion index 


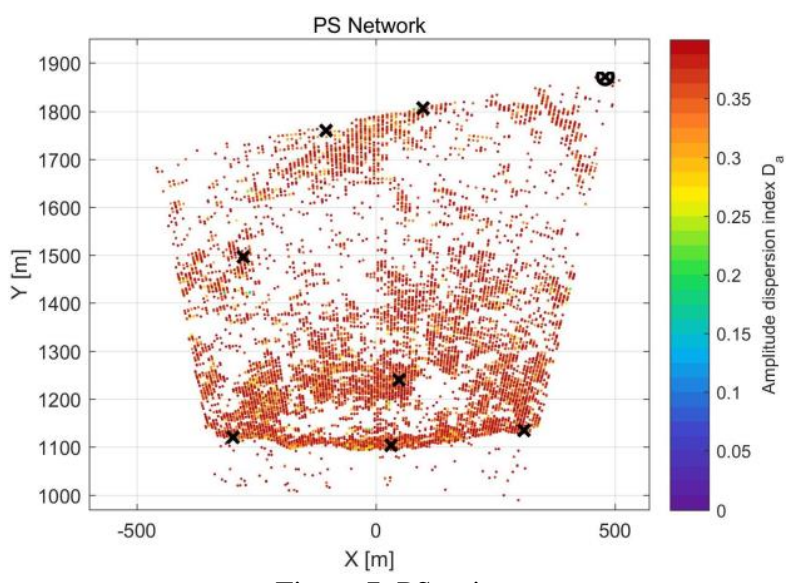

Figure 7. PS points

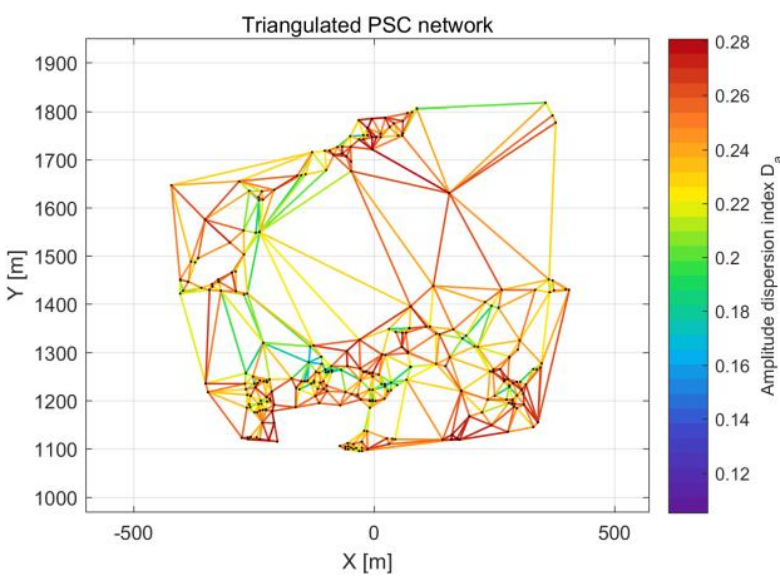

Figure 8. PS triangular mesh

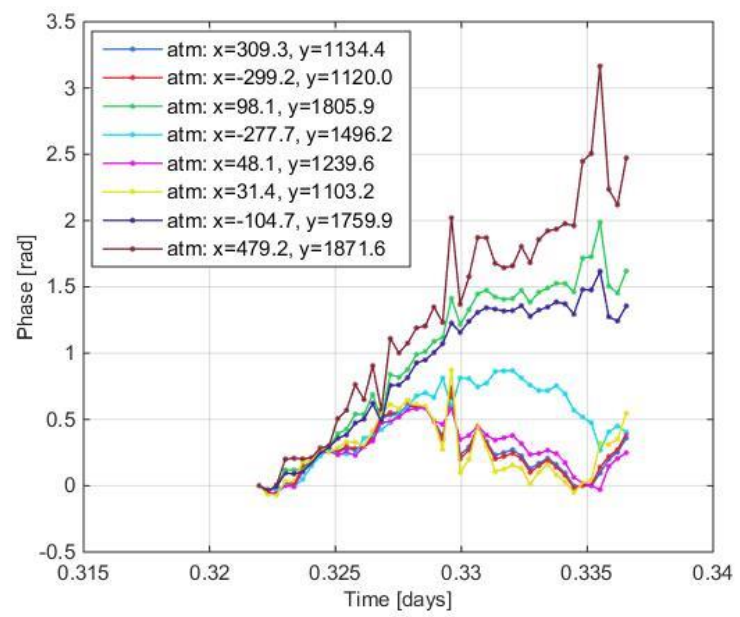

Figure 9. Atmospheric phase of PS points

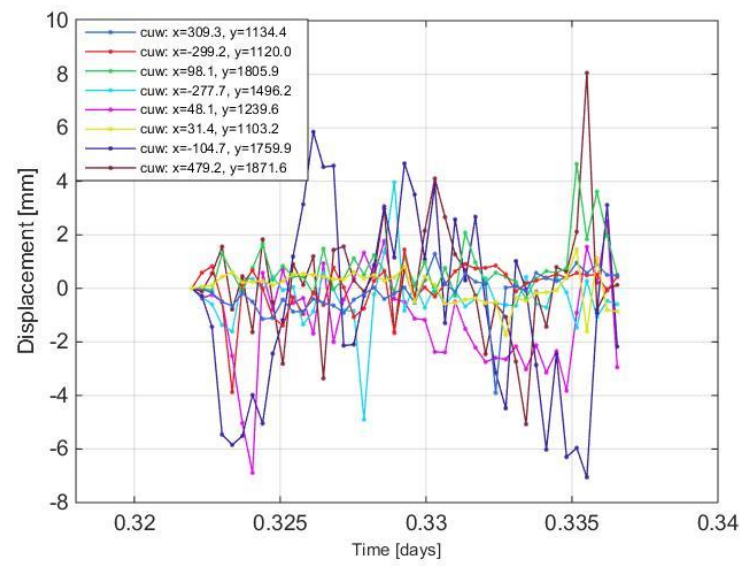

Figure 10. Deformation of PS points

\section{CONCLUSIONS}

Ma Liuzhuang landslide obtained by Fast-GBSAR is analysed in this paper. The results show that Fast-GBSAR can achieve accurate landslide deformation under severe weather conditions.

\section{ACKNOWLEDGEMENTS}

Thanks to the technical support provided by Metasensing BV and its Chinese partner, Beijing Five Star Electronic Technology Co., Ltd.

\section{REFERENCES}

Wang, P. and Zhou, X., 2012a. Ground-based SAR interferometry principles and its applications to displacement monitoring. Journal of Geomatics, 37(4), pp. 22-28.

Qu, C. W. and Wang, Y., 2008a. Research on synthetic aperture radar system based on frequency modulated continuous wave. Ship Electronic Engineering, 28(2), pp. 86-88.

Jiang, Z. H., Zhao Y., Huang, P. K., Wan, J. W. and Cheng, Y., 2008a. Research progress of SAR based on frequency modulated continuous wave. Modern Radar, 30(2), pp. 20-24.

Meta, A., Witj, J. M. and Hoogeboom, P., 2003. Time analysis and processing of FM-CW SAR. Proc IRS 2003, Dresden, Germany.

Qu, S. B., Wang, Y. P., Tan, W. X. and Hong, W., 2011a Deformation detection error analysis and experiment using ground based SAR. Journal of Electronics \& Information Technology, 33(1), pp. 1-7.

Wang, F., Wang, J. H. and Wu, Y., 1997a. Theory of differential radar interferometry and its application to deformation monitoring. Crustal deformation and earthquake, 17(3), pp. 63-67. 